\title{
Anticipated Changes in Pharmacy Practice by 2025: A Survey of Hospital Pharmacy Residents
}

\author{
Aurélie Guérin and Jean-François Bussières
}

\section{INTRODUCTION}

$\mathrm{O}$ ver the past decade, pharmacy practice has evolved considerably. ${ }^{1-5}$ The changes to pharmacy practice have resulted from expanded roles, changes in patients' expectations, introduction of new pharmacy practice models, and development of new technologies in the drug-use process.

To support this evolution, several professional societies have published vision statements inspired by the best available evidence. $^{6-11}$ For instance, in 2008 the Canadian Society of Hospital Pharmacists (CSHP) launched its "CSHP 2015" initiative, a vision of pharmacy practice excellence. The CSHP noted that "this quality care initiative strives to improve patients' medication-related outcomes and safety by advancing pharmacy practice excellence." 8 It was a patient-centred initiative that aimed to make the use of medications more effective (for inpatients and outpatients), more evidence-based, and safer (through policies, procedures, and use of technology) and to contribute meaningfully to public health.

Pharmacy students are tomorrow's generation of pharmacists, and it is important to adequately prepare them for current and future changes in pharmacy practice, to enable them to build a vision of pharmacy practice and be active in the development of their profession. Such exposure to pharmacy practice change starts during the undergraduate curriculum, with various teaching strategies and numerous contacts with different role models. Vision-building can also be reinforced throughout their careers through mentorship, participation in seminars or professional meetings, and readings.

Only limited literature has been published about the vision of pharmacy students regarding current and future pharmacy practice changes. The aim of this study was to survey pharmacy residents about the future of their profession to better prepare them to act within the profession.

\section{METHODS}

To identify current and future pharmacy practice changes, a literature search was conducted in the PubMed database using the following search strategy: (("changes"[All Fields] OR "future"[All Fields]) AND ("pharmacy"[All Fields] AND "practice"[All Fields]) OR "pharmacy practice"[All Fields] OR ("pharmacy"[All Fields] AND "practice"[All Fields]) OR "pharmacy practice"[All Fields]). Key pharmacy practice surveys and vision statements from US, Canadian, and European pharmacy societies were also considered. ${ }^{6-11}$

The authors, a pharmacy resident (A.G.) and a senior pharmacist (J.-F.B.) with 20 years' experience in hospital pharmacy management, held a brainstorming session to identify 4 change domains based on the results of the literature search.

The 4 change domains identified were academic pharmacy practice, general pharmacy practice, community pharmacy practice, and hospital pharmacy practice. Within these domains, a total of 48 statements about pharmacy practice change were developed. The statements were formulated in English and in French, with the objective of being explicit and measurable. Some of the terms in the statements were capitalized to increase clarity and to avoid confusion with other statements. The statements were formulated with the intention that not only Canadian pharmacy residents, but also European pharmacy residents would eventually be surveyed.

A paper version of the questionnaire was pretested with 4 pharmacy residents and 2 practising pharmacists for feasibility and clarity. Thereafter, a 48-question electronic version of the questionnaire was developed (using SurveyMonkey software, Palo Alto, California); this electronic version was pretested by 2 pharmacy residents for a targeted 20-min period to complete.

In the context of a 3-credit postgraduate hospital pharmacy management course offered in the faculties of pharmacy of the Université Laval (Quebec City, Quebec) and the Université de 
Montréal (Montréal, Quebec), all hospital pharmacy residents in the 2015/2016 cohort were contacted by e-mail in October 2015 and invited to respond to the survey. The study was realized in the academic context, and respondents were invited to complete the survey on their own time. The residents were told that participation was voluntary and anonymous and that the results of the survey might be published. Consent was obtained as part of regular teaching interactions with the students. All respondents gave oral consent before completing the survey.

Each respondent was asked to score the likelihood of occurrence of each pharmacy practice change by 2025 according to the following choices: 1 = very likely, $2=$ likely, 3 = unlikely, 4 = highly unlikely. An average score was calculated for each statement, to allow the statements to be ranked by likelihood. An average score greater than 3 (i.e., unlikely or highly unlikely) was considered to indicate a topic about which respondents most needed to be educated. Respondents were allowed to skip statements where they could not rate the likelihood. Only descriptive statistics were calculated.

\section{RESULTS}

Of the 62 hospital pharmacy residents in the 2 programs, 61 responded to the survey, for a $98 \%$ response rate. The respondents' demographic characteristics are presented in Table 1.

Of the 48 pharmacy practice changes covered in the survey, respondents considered 26 to be very likely or likely (i.e., average likelihood score < 2) (Table 2).

\section{DISCUSSION}

\section{The Future Is Now!}

With regard to academic pharmacy practice, respondents considered that all 6 changes were likely to occur by 2025. In particular, respondents believed that hospitals would require pharmacists to have formal postgraduate training to be hired, consistent with one of the objectives of the CSPH 2015 initiative (Objective 4.8, "100\% of new pharmacists entering hospital and related healthcare setting practice will have completed a Canadian Hospital Pharmacy Residency Boardaccredited residency"). ${ }^{8}$ Although $80 \%$ of active Quebec hospital pharmacists have a master of science postgraduate degree, this level of training is not widespread in the rest of the country. The survey responses indicate that pharmacy residents understand the need for postgraduate education to fulfill hospitals' and patients' expectations. Respondents also believed that knowledge and competency assessments would become widespread, both during pharmacy training and in practice. The entry-level PharmD implemented recently in Quebec (in 2007 at the Université de Montréal and in 2011 at the Université Laval) relies on competency assessment, and the inspection process of the Quebec regulatory authority (Ordre des
Table 1. Demographic Characteristics of Respondents

\begin{tabular}{|c|c|c|}
\hline Characteristic & $\begin{array}{r}\text { No. }(\%) \text { of } \\
(n\end{array}$ & $\begin{array}{l}\text { Respondents* } \\
=61)^{*}\end{array}$ \\
\hline Age (years) (median and IQR) & 24 & $(21-35)$ \\
\hline Sex & & \\
\hline Men & 22 & (36) \\
\hline Women & 39 & (64) \\
\hline Academic institution & & \\
\hline Université Laval & 21 & (34) \\
\hline Université de Montréal & 40 & (66) \\
\hline Area of practice desired $\dagger$ & & \\
\hline Community & 13 & $(21)$ \\
\hline Hospital & 59 & (97) \\
\hline University & 9 & (15) \\
\hline Other‡ & 1 & $(2)$ \\
\hline Experience in community pharmacy & & \\
\hline Has community experience & 61 & $(100)$ \\
\hline $\begin{array}{l}\text { Duration of experience (weeks) } \\
\text { (median and IQR) }\end{array}$ & 53 & $(8-900)$ \\
\hline $\begin{array}{l}\text { Experience in hospital pharmacy } \\
(n=60)\end{array}$ & & \\
\hline Has hospital experience & 55 & $(92)$ \\
\hline $\begin{array}{l}\text { Duration of experience (weeks) } \\
\text { (median and IQR) }\end{array}$ & 16 & $(7-280)$ \\
\hline $\begin{array}{l}\text { IQR = interquartile range. } \\
\text { *Except where indicated otherwi } \\
\text { †Respondents could select more } \\
\text { †Clinical research. }\end{array}$ & one opt & \\
\hline
\end{tabular}

pharmaciens du Québec), in place since 2010, also includes this approach. These program revisions have probably contributed to respondents' views that these changes are likely in the future. Respondents also considered inevitable the shift to continuous use of a professional portfolio. In Quebec, continuing education is encouraged, but no minimum threshold is required to keep one's licence to practice. Individual pharmacists can maintain a personal portfolio with documentation of all relevant activities undertaken, notwithstanding the accreditation of these activities by the regulatory authority. Finally, respondents considered likely the emergence of virtual teaching. Financial cuts in education, larger cohorts of pharmacy students over the past decade, and new expectations among pharmacy students regarding teaching modes and strategies may contribute to the emergence of more virtual teaching in pharmacy.

In terms of general pharmacy practice, respondents considered 8 of the 15 changes likely to occur. They believed that a majority of pharmacists would independently prescribe laboratory tests; independently prescribe, adjust, and renew drug therapy; and independently administer vaccines. Respondents were certainly aware of the recent expansion of pharmacists' scope of practice in Canada. They also believed that drug shortages would last at least until 2025. There is a growing body of literature about drug shortages everywhere in the world. Today's pharmacy residents were raised with social media, and 
This single copy is for your personal, non-commercial use only.

For permission to reprint multiple copies or to order presentation-ready copies for distribution, contact CJHP at cjhpedit@cshp.ca

\section{Table 2 (part 1 of 2). Likelihood of Occurrence of 48 Changes in Pharmacy Practice, According to Hospital Pharmacy Residents in Quebec}

Domain and Practice Change Statement*

\section{Academic pharmacy practice ( $n=6$ statements)}

Credentials: Hospitals will require pharmacists to have completed formal postgraduate training to be hired and work within the hospital

Academic programs: A majority of the evaluation conducted during an academic pharmacy curriculum will be based not only on knowledge but also on abilities and competencies

Professional portfolio: Pharmacists will have to document their continuing education in a portfolio on the website of a regulatory authority

À la carte training: Pharmacists will be required to complete "à la carte" online advanced training to be allowed to perform certain activities

Competency evaluation: A majority of pharmacists will be evaluated periodically to assess their competencies

Virtual teaching: A majority of academic clinical patient cases during undergraduate and postgraduate training will be taught using virtual simulations rather than real patients

\section{General pharmacy practice ( $\boldsymbol{n}=15$ statements)}

Prescribing of laboratory tests: Pharmacists will be allowed to independently PRESCRIBE laboratory tests Prescribing of drugs: Pharmacists will be allowed to independently ADJUST drug therapy Prescribing of drugs: Pharmacists will be allowed to independently RENEW drug therapy Procurement of medicines: A majority of pharmacists will frequently encounter drug shortages Prescribing of drugs: Pharmacists will be allowed to independently INITIALLY PRESCRIBE drug therapy Social media: A majority of pharmacists will have a presence on the web and be active in blogging Outcome-based practice: A majority of pharmacists will organize and prioritize their practice on the basis of patient outcomes (i.e., evidence-based pharmacy)

Vaccine administration: Pharmacists will be allowed to independently ADMINISTER vaccines Regulation of technicians: Pharmacy technicians will be regulated by the pharmacy regulatory authority and will have a say in the evolution of pharmacy practice

Specialized channels: A majority of new drugs commercialized in a given market will be launched and publicized for professionals and patients through specialized channels, to avoid any delays in knowledge transfer

Patient-pharmacist communication: A majority of patients will interact with their pharmacists by email (for questions and follow-up)

Patient compliance: A majority of patients will use applications on their smartphones to document their adherence to drug therapy, and these data will be synchronized with pharmacy information systems for online monitoring of adherence

Pharmacogenomics: A majority of patients will have their genomes mapped early in life and pharmacists will have access to these results to adjust drug therapy on the basis of known polymorphisms (to avoid side effects or optimize expected drug effects)

Patients' use of smartphone technology: A majority of patients will use their smartphones to record conversations with a pharmacist, to allow quiet listening once at home

Patients speaking up: Patients will be allowed to blog on PubMed (similar to PubMed Commons for professionals/authors) and to comment on published articles

\section{Community pharmacy practice ( $n=4$ statements)}

Rate your pharmacist: A majority of pharmacists will have been rated by a significant number of patients on "rate your pharmacist" websites

Internet community pharmacy: A majority of patients will buy their outpatient/home medication through the Internet, while retaining the option of going to the related/affiliated retail pharmacy Reimbursement: A majority of outpatient/home-based drug prescriptions will be reimbursed by third-party payers only if they are processed through online pharmacies (i.e., Internet pharmacies) Community pharmacy ownership: A majority of community pharmacies will be owned by nonpharmacists

Average Likelihood Scoret (No. of Respondents)

$1.35(n=48)$

$1.41(n=39)$

$1.62(n=50)$

$1.63(n=43)$

$1.72(n=36)$

$1.93(n=57)$ $1.17(n=29)$

$1.20(n=30)$

$1.27(n=29)$

$1.34(n=58)$

$1.43(n=30)$

$1.74(n=59)$

$1.91(n=54)$

$1.98(n=59)$

$2.12(n=60)$

$2.48(n=60)$

$2.55(n=60)$

$2.57(n=61)$

$2.70(n=61)$

$2.82(n=61)$

$3.15(n=61)$ respondents believed that pharmacists will have an active presence on the web in the future. Finally, respondents believed that a majority of pharmacists will organize and prioritize their practice on the basis of patient outcomes. This is certainly consistent with the teaching of evidence-based approaches in academic institutions and faculties of pharmacy.
In terms of community pharmacy practice, respondents considered none of the 4 changes likely to occur by 2025 .

Finally, with regard to hospital pharmacy practice, respondents considered 12 of the 23 changes likely to occur by 2025 . Respondents believed that they would be working in a consultation mode and in collaboration with other professionals and 


\section{Table 2 (part 2 of 2). Likelihood of Occurrence of 48 Changes in Pharmacy Practice, According to Hospital Pharmacy Residents in Quebec}

Domain and Practice Change Statement*

Hospital pharmacy practice ( $\boldsymbol{n}=23$ statements)

Collaborative practices: A majority of hospital pharmacists will work in collaboration with other professionals

Documentation of practices: A majority of hospital pharmacists will document the pharmaceutical

plan in the patient's file

Robots: A majority of hospital doses dispensed will be prepared through automated systems

Compounding of drugs: A hospital pharmacy department will have to be certified by a national regulatory authority to be allowed to compound drugs for patients within the hospital

Tech check tech: A majority of hospital drug distribution/dispensing activities will be performed by pharmacy technicians with independent checking by another pharmacy technician

Traceability: A majority of drug doses dispensed will be traceable through bar codes used at patients' bedsides

Pharmacists' accountability: Hospital pharmacists will be held accountable for evaluating and monitoring patients' outcomes following their activities and interventions

Certification: A majority of hospital pharmacy technicians will be locally or nationally certified to be allowed to perform tasks, including "tech check tech"

Traceability: A majority of drug doses compounded by the hospital pharmacy will be traceable through imaging

Teaching of drug administration: Hospital pharmacists will be allowed to independently TEACH subcutaneous administration

Consultation mode: A majority of patients followed by a hospital pharmacist will be identified following a medical consultation request

Pharmacist-physician communication: A majority of hospital pharmacists will use smartphones to interact with physicians to clarify drug prescriptions and drug-related problems

Features of electronic health records: A majority of patients will get access to advanced features of electronic health records to participate actively to their own care

Telepharmacy drug distribution: A majority of hospital pharmacists will validate drug orders from a distant site

Prioritization: A majority of hospital pharmacists' daily workload will be determined by software

that will identify/target relevant patients based on a higher probability of drug-related problems

Pharmacists at the bedside: A majority of hospital pharmacists will spend all of their time at patients' bedsides or in patient care programs

Medication errors: A majority of drug errors occurring within a hospital will be reported in a national registry that patients and all stakeholders will be allowed to monitor and consult

Telepharmacy clinical services: A majority of hospital pharmacy departments will be equipped

or have access to a telepharmacy infrastructure to treat and follow patients at distance

Pharmacy dispensing: A majority of hospital pharmacy departments will operate without any pharmacists, using only pharmacy technicians for drug dispensing

Clinical pharmacy activities: A majority of clinical pharmacy activities will be performed by clinical nurses

(e.g., medication reconciliation)

Lawsuits: A majority of hospital pharmacy departments will have been sued by patients for medication errors

Pharmacist remuneration: Hospital pharmacists will be paid on the basis of the number of professionals activities performed

Hospital pharmacy leadership: A majority of hospital pharmacy departments will be managed by nonpharmacists

*Capitalization of certain terms was intended to increase clarity of the statement and avoid confusion with other similar statements. tScores: 1 = very likely, 2 = likely, $3=$ unlikely, 4 = highly unlikely. Within each section, the statements are ranked by ascending order of average score (i.e., descending likelihood).

that they would have to document their pharmaceutical plans in patients' files. They also considered likely the use of robots, the traceability of doses through bar codes used at the patients' bedside, the traceability of compounded drug doses through imaging, the certification of pharmacy compounding facilities and pharmacy technicians, and the use of smartphones to interact with physicians. Moreover, they considered that
Average Likelihood Scoret (No. of Respondents)
$1.06(n=34)$
$1.09(n=33)$
$1.19(n=42)$
$1.37(n=40)$
$1.40(n=45)$
$1.47(n=55)$
$1.52(n=46)$
$1.59(n=49)$
$1.67(n=48)$
$1.74(n=43)$
$1.82(n=50)$
$1.93(n=60)$
$2.08(n=59)$
$2.10(n=60)$
$2.13(n=60)$
$2.22(n=54)$
$2.24(n=54)$
$2.34(n=59)$
$2.35(n=55)$
$2.63(n=59)$
$2.83(n=59)$
$3.12(n=59)$
$3.19(n=60)$ 


\section{A Need for Reflection and Action}

On the basis of our own knowledge and perspectives, we believe that all of the pharmacy practice changes proposed in this survey are realistic and will affect pharmacy by the year 2025. However, the hospital pharmacy residents surveyed considered just over half of these changes very likely or likely by 2025. A few factors can explain this discrepancy in perceptions. Most of the statements were worded using the term "a majority of...", and it is possible that respondents were aware of the emergence of many of these pharmacy practice changes but did not believe that a majority of pharmacists or patients would be in the particular situation by 2025 . Only 4 statements had an average likelihood score of 3 or higher, indicating changes about which pharmacy residents seemed to need the most education: these statements related to the management of hospital pharmacy departments, the ownership of community pharmacies, payment of pharmacists according to the number of professional activities performed, and patients' expression of their views about published articles. Finally, changes within a profession take time. In particular, previous work from the authors' research group has indicated that it takes a fair amount of time to change practices in the field of pharmacy. ${ }^{12}$ Respondents may have been aware of the significant time required to implement changes in a profession.

Given these results, it appears reasonable to increase the awareness of hospital pharmacy residents about future pharmacy practice changes and to give them the knowledge and tools to cope with the changes, thus allowing them to be better prepared to adapt over the course of their careers. These results can be used for discussions within hospital pharmacy residency curricula through journal clubs. They could also be used for case-based learning, in leadership programs, and in other mentorship activities. ${ }^{13-15}$ We believe that this questionnaire and its results can contribute to the debate about the future of pharmacy practice. The questionnaire could also be administered to other groups (e.g., undergraduate students, practising pharmacists, and pharmacy technicians).

\section{Limitations}

This survey study had some limitations. The pharmacy practice changes listed in the survey were identified by a pair of investigators, one pharmacy resident and one pharmacist, although the survey itself was pretested by a heterogeneous pilot group of pharmacy students. Both of the authors specialize in hospital pharmacy, so the statements for community practice are not as robust as those for hospital practice. The 4 domains chosen for the survey - academic, general pharmacy, community pharmacy, and hospital pharmacy — were not validated but seem to cover the full scope of pharmaceutical practice. Moreover, the decision to classify the statements by domain, instead of treating them all as general (and academic) is a significant limitation. Indeed, it assumes that hospital practice is fundamentally different from community practice in many ways, whereas the differences between these areas of practice are probably related more to hospital practice being just a bit ahead in making changes, with community pharmacies likely to make the same shifts over time. The statements were presented to the pharmacy residents without further detail or background. Some statements may have been misinterpreted, given residents' limited background in pharmacy practice at this stage in their careers. Respondents were allowed to skip statements for which they could not rate likelihood. Only half of the respondents (or fewer) responded to the 4 statements related to prescribing; we are unable to explain these low response rates. This survey was a pilot test, and other groups should be surveyed about the same topics before any meaningful or major conclusions can be reached.

\section{CONCLUSION}

This study has presented the perceptions of 61 Canadian hospital pharmacy residents about 48 potential pharmacy practice changes. Respondents considered that 26 of the 48 changes were very likely or likely to occur by 2025 in Canada. Hospital pharmacy residents should be aware of current and future pharmacy practice changes, so they can be better prepared to act within the profession. Pharmacy residency programs should incorporate discussions about future pharmacy practice changes to help increase preparedness among students. Additional work is needed to better understand perceptions about future pharmacy practice changes. A survey of practising pharmacy professionals, with different durations of work experience, could complement this survey.

References

1. Scheckelhoff DJ. Fifty years of advancement in American hospital pharmacy. Am J Health Syst Pharm. 2014;71(22):1947-57.

2. 2015 EAHP statements survey. Brussels (Belgium): European Association of Hospital Pharmacists; 2016 [cited 2016 Feb 3]. Available from: www.eahp.eu/publications/survey/content/2015-survey

3. EAHP survey 2010: hospital pharmacy practice in Europe. Brussels (Belgium): European Association of Hospital Pharmacists; 2016 [cited 2016 Feb 3]. Available from: www.eahp.eu/publications/survey/eahp2010-survey-hospital-pharmacy-practice-europe

4. Bussières JF, Bonnici A, Tanguay C. Perspective québécoise et canadienne de la pratique pharmaceutique hospitalière 2013-2014. Pharmactuel. 2015;48(Suppl 2):1-41.

5. Guérin A, Lebel D, Marando N, Prot-Labarthe $S$, Bourdon O, Bussières JF. [Determinants of the evolution of hospital pharmacy in France and Quebec: perception of hospital pharmacists]. Ann Pharm Fr. 2014;72 (3):202-15. Article in French.

6. 2020 vision: FIP's vision, mission and strategic plan. The Hague (Netherlands): International Pharmaceutical Federation; [cited 2016 Feb 3]. Available from: https://www.fip.org/files/fip/strategic $\% 20$ plan $\% 20$ no $\%$ 20annexes.pdf

7. 2015 initiative. Bethesda (MD): American Society of Health-System Pharmacists; 2016 [cited 2016 Feb 3]. Available from: http:// www.ashp.org/2015 
8. CSHP 2015 - targeting excellence in pharmacy practice. Ottawa $(\mathrm{ON})$ : Canadian Society of Hospital Pharmacists; 2016 [cited 2016 Feb 3]. Available from: www.cshp.ca/cshp2015/index_e.asp

9. The European statements of hospital pharmacy. Eur J Hosp Pharm 2014;21(5):256-8.

10. American College of Clinical Pharmacy. Standards of practice for clinical pharmacists. Pharmacotherapy. 2014;34(8):794-7.

11. Blueprint for pharmacy. Ottawa $(\mathrm{ON})$ : Canadian Pharmacists Association; 2016 [cited 2016 Feb 3]. Available from: www.pharmacists.ca/index.cfm/ pharmacy-in-canada/blueprint-for-pharmacy/

12. Guérin A, Hall K, Bussières JF. Pharmacy practices and technologies: evidence for effectiveness and adoption into Canadian hospital pharmacy practice. Can J Hosp Pharm. 2015;68(6):474-7.

13. Thistlethwaite JE, Davies D, Ekeocha S, Kidd JM, MacDougall C, Matthews $\mathrm{P}$, et al. The effectiveness of case-based learning in health professional education. A BEME systematic review: BEME Guide No. 23. Med Teach. 2012;34(6):e421-44.

14. Wallace $S$. Impact of a student leadership development program. Am J Pharm Educ. 2014;78(4):86.

15. Chesnut R, Tran-Johnson J. Impact of a student leadership development program. Am J Pharm Educ. 2013;77(10):225.
Aurélie Guérin, DPharm, is a Collaborator with the Pharmacy Practice Research Unit and the Pharmacy Department, Centre hospitalier universitaire Sainte-Justine, Montréal, Quebec. She is also a Research Assistant in the Academic Practice Unit, Birmingham Children's Hospital, Birmingham, United Kingdom.

Jean-François Bussières, BPharm, MSC, MBA, FCSHP, is Director of the Pharmacy Practice Research Unit and the Pharmacy Department, Centre hospitalier universitaire Sainte-Justine, Montréal, Quebec. He is also a Clinical Professor with the Faculty of Pharmacy, Université de Montréal, Montréal, Quebec.

Competing interests: None declared.

\section{Address correspondence to:}

Jean-François Bussières

Pharmacy Department

Centre hospitalier universitaire Sainte-Justine

3175, chemin de la Côte Sainte-Catherine

Montréal QC H3T 1C5

e-mail: jf.bussieres@ssss.gouv.qc.ca

Funding: None received.

\section{CSHP's 2017 Professional Practice Conference Keynote Speaker: Timothy Caulfield}

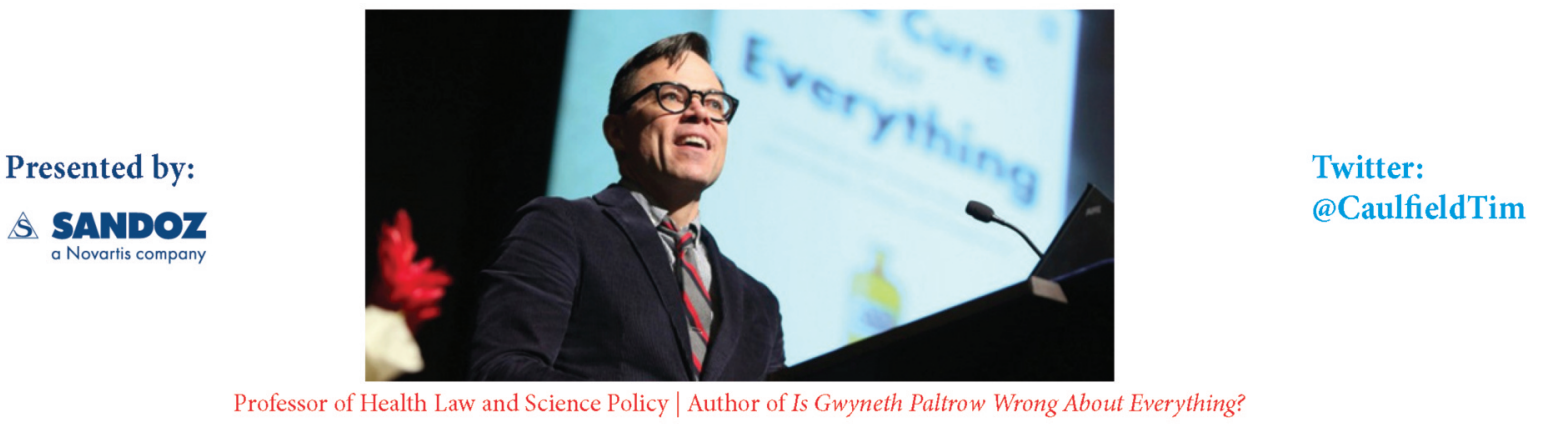

Professor Timothy Caulfield is an unrivaled communicator who debunks myths and assumptions about innovation in the health sector-from research on stem cells to diets to alternative medicine-for the benefit of the public and decision-makers. He is a Canada Research Chair in Health Law and Policy, and a Professor in the Faculty of Law and the School of Public Health at the University of Alberta. He has been the Research Director of the Health Law Institute at the University of Alberta since 1993.

He writes frequently for the popular press on a range of health and science policy issues and is the author of The Cure for Everything: Untangling the Twisted Messages about Health, Fitness and Happiness, and his most recent book, Is Gwyneth Paltrow Wrong About Everything?: When Celebrity Culture and Science Clash. 